\title{
Helium Segregation to Screw and Edge Dislocations in $\alpha$-Iron and their Yield Strength
}

\author{
Enrique Martínez, ${ }^{1, *}$ Daniel Schwen, ${ }^{1}$ and Alfredo Caro ${ }^{1}$ \\ ${ }^{1}$ Material Science and Technology Division, MST-8, \\ Los Alamos National Laboratory, Los Alamos, 87545 NM, USA
}

(Dated: October 21, 2014)

\begin{abstract}
Helium (He) presents one of the mayor concerns in the nuclear materials community as it modifies the mechanical properties of the system withstanding fast neutron spectra promoting swelling and embrittlement. Ferritic/martensitic steels are one of the main candidates as structural materials for future nuclear applications. Experimentally the bubble distribution is observed to vary depending on irradiation conditions (temperature, dose rate and total dose). However, traditional atomistic models decouple the role of temperature in the mechanical properties from its effect on the bubble distribution. In this paper we study substitutional He segregation to screw and edge dislocations in $\alpha$-Fe at different temperatures. We use an object kinetic Monte Carlo methodology to obtain general trends in bubble distribution and a canonical Monte Carlo algorithm, with full atomistic fidelity, to find the He distribution at the dislocation cores. Molecular dynamics have subsequently been applied to study the yield strength, which increases significantly in the presence of He, more remarkably for the edge dislocation. The total stress fits a Kocks relation. However, if the lattice resistance is subtracted, the relation between the critical shear stress and the temperature results non-monotonic for the screw character. To reproduce this effect, we propose to modify Kocks' relation adding a second order term in temperature that extends the range of applicability of the model.
\end{abstract}

PACS numbers: 61.80.Az,61.72Cc,61.72.Ji

Keywords: Helium; Segregation; Dislocation Mobility; Modeling; Mote Carlo

\section{INTRODUCTION}

The presence of Helium (He) in next generation nuclear environments ${ }^{1-3}$ poses great material challenges as it precipitates in the form of bubbles, and usually segregates into dislocations and grain boundaries, promoting swelling, hardening and embrittlement, which is ultimately the main failure mechanism occurring in these materials $^{4-7}$. The distribution of bubble diameters and spacing between them is controlled by the irradiation conditions (temperature, dose rate and total dose) and the material properties (mobilities and energetics).

He effects have been lengthily studied both experimentally and theoretically. On the experimental front, Kramer et al. ${ }^{4}$ studied the effect of He in the mechanical properties of 304 stainless steels. They observed a severe loss of ductility rationalized in terms of He segregation to carbide particles at grain boundaries that enhanced void formation and cracking. More recent results on $\mathrm{He}$ implantation show a strong embrittlement effect in different steels $^{8-15}$ even for small He contents. This deterioration of the mechanical properties is associated to He segregation to extended defects. Insight in this matter has been provided by theoretical models. Rate theory has been used to study bubble formation and bubble to void transition that promotes swelling ${ }^{16-18}$. Although useful in several aspects, rate theory needs an extended list of parameters, usually obtained either from experiments or lower scales computational models. This is one of the main reasons why classical Molecular Dynamics (MD) simulations have become so prominent.

There has been a number of atomistic studies focused on the interaction of dislocations with preexistent $\mathrm{He}$ bubbles and the energetics of He at dislocations or grain boundaries in $\mathrm{Fe}^{19-25}$. However, the combined study of both, He segregation into dislocations, and how this segregation modifies the yield strength of the system is not available in the literature, even though this case-scenario is physically more meaningful. That is, for a given material and an irradiation dose rate, temperature determines the bubble distribution on dislocations, varying in diameter and spacing. This effect sums up to the fact that the yield stress for a dislocation to overcome a fixed distribution of obstacles also depends on temperature, and therefore, both processes are convoluted. Understanding this coevolution is the purpose of this work, in which we use both object kinetic Monte Carlo (OKMC) and canonical Metropolis Monte Carlo (CMC) in the Kawasaki dynamics formalism, to obtain different bubble distributions as a function of temperature, dose rate and dislocation character. We then study the mechanical response of these decorated dislocations and obtain an analytical expression for the increase of yield stress induced by He.

\section{RESULTS}

The study of the real dynamics of He segregation to screw and edge dislocations in $\alpha$-Fe and the resultant dislocation mobility is challenging. MD calculations are limited in simulated time and therefore it is difficult to extract meaningful physics from them. Kinetic Monte Carlo algorithms have been proposed to try to overcome the MD time scale problem ${ }^{26-30}$, however, in the case of He hopping in the presence of dislocations, the existence of complex mechanisms and low barriers decreases 
dramatically their efficiency. This study focuses on the bubble distribution at a given temperature and how the temperature modifies the spacing between bubbles and the bubble diameters rather than in the actual dynamics of the process. Consequently, we use two different Monte Carlo algorithms, an OKMC to study general trends in the distribution of bubbles and a CMC method ${ }^{31}$ with a fix rate of transmutation of Fe into He that mimics the behavior of $\mathrm{He}$ in the presence of a dislocation field. This algorithm has been implemented in the MD code LAMMPS $^{32}$, obtaining a very powerful hybrid MC-MD scheme that takes into account chemical mixing, structural relaxations, and thermal vibrations.

\section{A. General trends for bubble diameter and spacing depending on temperature and dose rate}

To obtain general trends of the bubble distributions on a linear sink, the OKMC algorithm ${ }^{33}$ has been used. The main goal is to convince the reader that the bubble distribution depends on both temperature and dose rate. For that, a quasi-1D model on an underlying bcc lattice with 8192 sites $(2 \times 2 \times 1024)$ has been setup with He atoms diffusing to first nearest neighbors with a migration barrier of $E_{m}=0.8 \mathrm{eV}$ and a prefactor of $\nu=10^{12} \mathrm{~s}^{-1}$. He atoms are created at random at a prescribed dose rate. Defects interact to form clusters with an infinite binding energy, i.e. when a cluster forms it will not dissolve (mimicking the large He-He binding energy). Runs have been carried out at different temperatures and dose rates until the fraction of defects in the sample was 0.25 . Results are shown in Fig. 1, where the evolution of the bubble diameter (D) and spacing (L) between bubbles are plotted. Both D and L are monotonically increasing functions of the temperature. Temperature increases the defect mobility for a given dose rate, which increases the reaction probability and therefore the propensity for bubble nucleation. We also notice that the lower the dose rate the larger the bubble diameter and therefore larger the spacing between bubbles. For a given temperature, increasing the dose rate decreases the bubble nucleation probability.

The evolution of the bubble diameter was fitted with functions of the simple form: $D=D_{0}+\alpha \cdot\left(T-T_{0}\right)^{\beta}$. The diameter has been obtained, assuming sphericity, using the relation $\langle N\rangle \Omega=\frac{1}{6} \pi D^{3}$, where $\langle N\rangle$ is the average number of particles in a cluster and $\Omega$ is the atomic volume. The total number of defects in the sample is known, and therefore, a relation between the diameter and the spacing between bubbles can be derived: $L=\frac{D^{3} \pi L_{T}}{6 \Omega N_{T}}$, with $L_{T}$ the total length of the sample and $N_{T}$ the total number of defects. General trends are therefore obtained following this methodology.

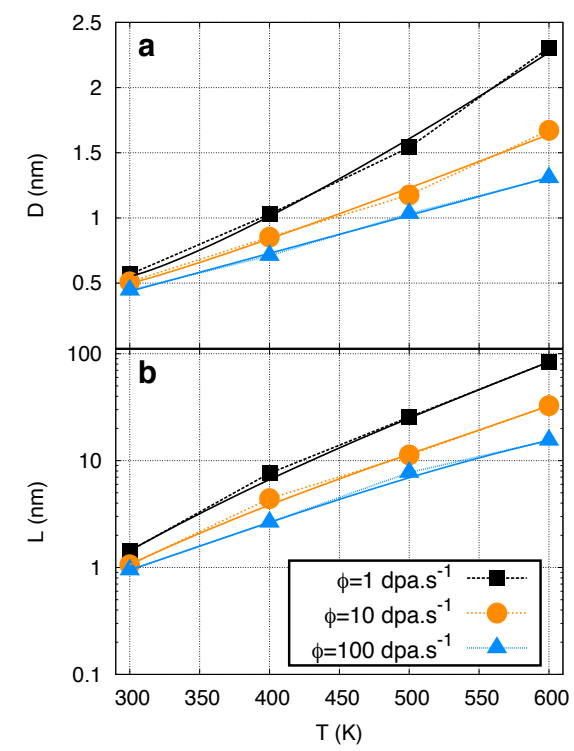

FIG. 1: (Color online) OKMC results for the evolution depending on temperature and dose rate of (a) $\mathrm{He}$

bubble diameter and (b) He bubble spacing.

TABLE I: Fitting parameters for the bubble diameter $\left(D=D_{0}+\alpha \cdot\left(T-T_{0}\right)^{\beta}\right) . T_{0}=300 K$ in all cases.

\begin{tabular}{lccc}
\hline & $D_{0}(\mathrm{~nm})$ & $\alpha$ & $\beta$ \\
\hline \hline$\phi=1 \mathrm{dpa} . \mathrm{s}^{-1}$ & 0.55 & 0.018 & 1.2 \\
$\phi=10 \mathrm{dpa}^{-1} \mathrm{~s}^{-1}$ & 0.50 & 0.021 & 1.1 \\
$\phi=100 \mathrm{dpa}^{-1} \mathrm{~s}^{-1}$ & 0.44 & 0.029 & 1.0 \\
\hline
\end{tabular}

\section{B. He segregation to screw and edge dislocations} as determined by canonical Monte Carlo

Two samples containing a screw and an edge dislocation respectively were generated using linear elasticity ${ }^{34}$.

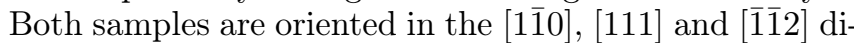
rection and fix boundary conditions in the [110] direction and periodic in the other two were applied. The Burgers vector of both dislocations is the $\frac{a_{0}}{2}[111]$, where $a_{0}$ is the lattice parameter of $\alpha-\mathrm{Fe}^{35}$. The dislocation line for the screw case is along the [111] direction while for the edge the dislocation line lies along the $[\overline{1} \overline{1} 2]$ direction. Two layers of $12 \AA$ each were fixed in the upper and lower region of the simulation box. The Fe-Fe interatomic potential given by Ackland et al. ${ }^{36}$, the He-He interaction by Beck et $a l .{ }^{37}$ and the Fe-He by Juslin et al. ${ }^{38}$ were used in both MD and CMC simulations. Three temperatures have been considered, $100 \mathrm{~K}, 300 \mathrm{~K}$ and $500 \mathrm{~K}$. The He atoms were inserted in the system at the expense of Fe atoms, i. e., transmuting Fe into $\mathrm{He}$ at a prescribed $\mathrm{CMC}$ rate. In this way, substitutional He atoms were 
introduced every $8000 \mathrm{CMC}$ steps. To sample the vibrational degrees of freedom $500 \mathrm{MD}$ steps were performed between CMC steps. This methodology leads to bubbles with a He-to-vacancy ratio close to one, similar to the calculated equilibrium value ${ }^{35,39}$. The CMC stopped when a total of $100 \mathrm{He}$ atoms were inserted in the sample, which represents a concentration per dislocation unit length of $c_{H e}^{\text {screw }}=3.68 \mathrm{~nm}^{-1}$ and $c_{H e}^{\text {edge }}=3.57 \mathrm{~nm}^{-1}$ for the screw and edge dislocations, respectively.

\section{He segregation to screw dislocations}

Dislocations are preferential sites for heterogeneous nucleation of He bubbles. The thermodynamic driving force for a substitutional He atom to be attracted towards the dislocation is given by the gradient of chemical potential. At $0 \mathrm{~K}$ this gradient can be estimated calculating the interaction energy. This interaction energy is defined $\operatorname{as}^{40}$ :

$$
\mathcal{E}_{\mathrm{He}-\mathrm{D}}^{\text {int }}=\mathcal{E}[\mathrm{He}-\mathrm{D}]+\mathcal{E}[\mathrm{Fe}]-\{\mathcal{E}[\mathrm{D}]+\mathcal{E}[\mathrm{He}]\}
$$

where $\mathcal{E}[\mathrm{He}-\mathrm{D}]$ is the energy of the system with the substitutional $\mathrm{He}$ at the dislocation core, $\mathcal{E}[\mathrm{Fe}]$ is the energy of the pure Fe system taken as reference, $\mathcal{E}[\mathrm{D}]$ is the energy of the system with the dislocation and $\mathcal{E}[\mathrm{He}]$ is the energy of the Fe system with one substitutional He. We have obtained a value of $\mathcal{E}_{\mathrm{He}-\mathrm{D}}^{i n t}=-0.28 \mathrm{eV}$ for the screw dislocation. Monte Carlo simulations were performed to obtain the He segregation distribution in a system with a screw dislocation for the three temperatures of interest (see Fig. 2). The sample dimensions were $16.2 \times 27.2$ $\times 14 \mathrm{~nm}$. At $100 \mathrm{~K}$, the average number of He atoms per bubble is 10 , which leads to an average diameter of around $0.59 \mathrm{~nm}$ for a $c_{H e}=3.68 \mathrm{~nm}^{-1}$ and an average spacing between bubbles of $2.72 \mathrm{~nm}$. The average diameter at $300 \mathrm{~K}$ is about $0.62 \mathrm{~nm}$ with a bubble spacing of $3.02 \mathrm{~nm}$ and at $500 \mathrm{~K}$ the average diameter is about $0.67 \mathrm{~nm}$, which leads to a bubble spacing of $3.88 \mathrm{~nm}$. The bubbles can be considered spherical at every temperature since surface tension becomes the dominant term in the energetics of the system.

\section{He segregation to edge dislocations}

The interaction energy of a substitutional He atom at the core of the edge dislocation is $\mathcal{E}_{\mathrm{He}-\mathrm{D}}^{i n t}=-0.5 \mathrm{eV}$, which results in a strong thermodynamic driving force for the He to segregate at the dislocation core. This interaction energy is more attractive than for the screw dislocation and thus we expect a stronger pinning effect for the edge character.

As it was done for the screw dislocation, we have studied substitutional He segregation into the edge dislocation core using the CMC methodology. Figure 3 shows

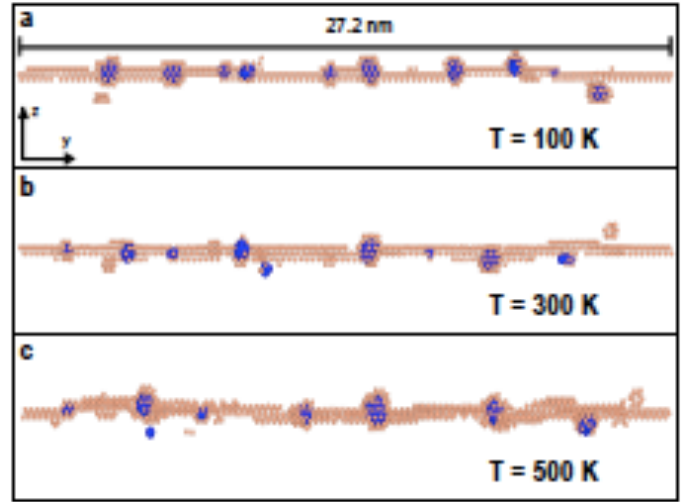

FIG. 2: (Color online) He bubble distribution at a screw dislocation core in $\alpha$-Fe. Light brown atoms represent non-bcc Fe atoms according to the common neighbor analysis while $\mathrm{He}$ atoms are depicted in blue

(a) $T=100 \mathrm{~K}$; (b) $T=300 \mathrm{~K}$ and; (c) $T=500 \mathrm{~K}$

the bubble configuration at the dislocation core for the temperatures studied. The sample dimensions were 16.3 $\times 9.78 \times 28 \mathrm{~nm}$. At $\mathrm{T}=100 \mathrm{~K}$ the average bubble diameter was $0.51 \mathrm{~nm}$ with an average spacing between bubbles of $2 \mathrm{~nm}$. When the temperature was increased to $300 \mathrm{~K}$, the average bubble diameter became $0.64 \mathrm{~nm}$ and the bubble spacing $3.5 \mathrm{~nm}$. Finally, for $500 \mathrm{~K}$ we found an average diameter of $0.77 \mathrm{~nm}$ and an average spacing of $6.29 \mathrm{~nm}$. For all temperatures again the bubble structure can be considered spherical.

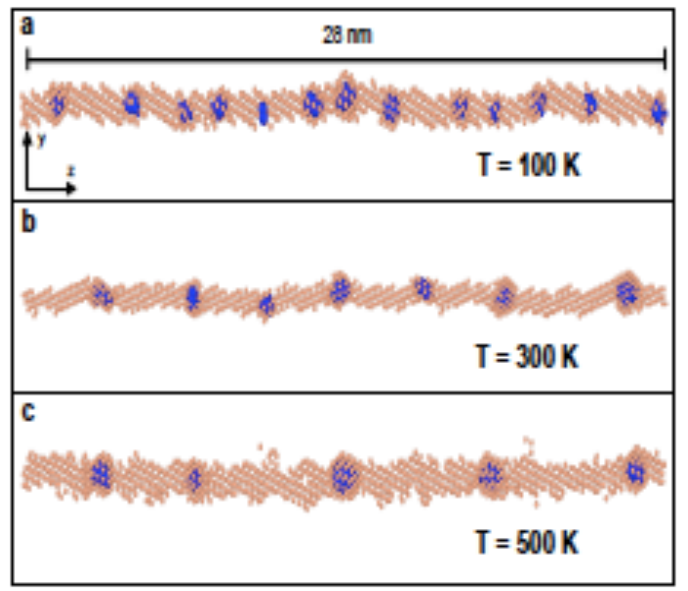

FIG. 3: (Color online) He bubble distribution at an edge dislocation core in $\alpha$-Fe. Light brown atoms represent non-bcc Fe atoms according to the common neighbor analysis while $\mathrm{He}$ atoms are depicted in blue

(a) $T=100 \mathrm{~K}$; (b) $T=300 \mathrm{~K}$ and; (c) $T=500 \mathrm{~K}$

Figure 4 shows the segregation behavior using both Monte Carlo approaches. The resulting trends are similar and can be described by the relation $D=D_{0}+\alpha \cdot(T-$ $\left.T_{0}\right)^{\beta}$, with $T_{0}=100$, and $D_{0}=0.59 \mathrm{~nm}, \alpha=6.05 \cdot 10^{-5}$ and $\beta=1.2$ for the screw dislocation and $D_{0}=0.51 \mathrm{~nm}$, 
$\alpha=6.5 \cdot 10^{-4}$ and $\beta=1.0$ for the edge dislocation. The function is close to linear, with a crossover that correlates to the relation of the substitutional He binding energy to the dislocation cores and the He binding energies to the bubbles themselves. The spacing between bubbles as obtained from CMC is shown in Fig. 4[b]. For the screw dislocation we see that the points follow accurately the fitting function. On the other hand, for the edge dislocation there is a slight deviation from the predicted values, probably due to lack of better statistics and the fact that the binding energies between $\mathrm{He}$ was not proper in the OKMC model.

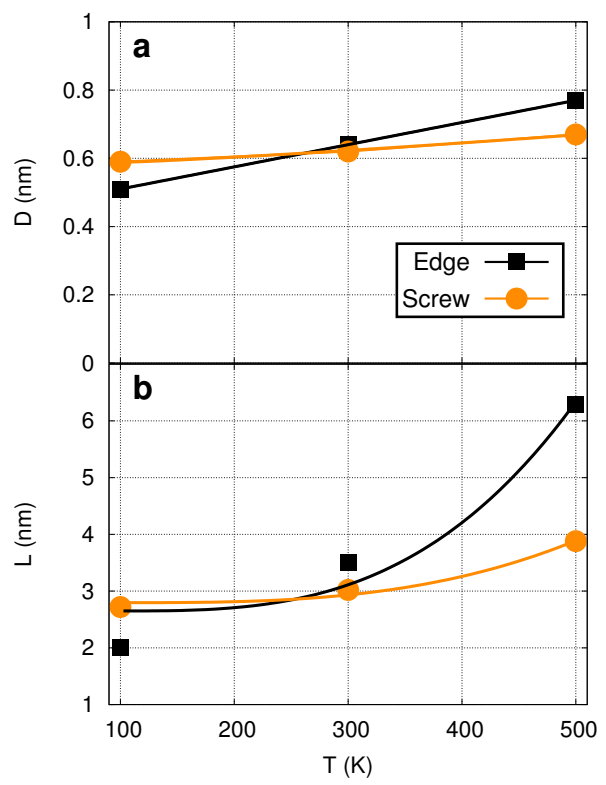

FIG. 4: (Color online) Canonical MC results for He bubbles (a) diameter and; (b) spacing. Points are fitted to the expression obtained from OKMC calculations, i.e. $D=D_{0}+\alpha \cdot\left(T-T_{0}\right)^{\beta}$ and $L \propto D^{3}$ (solid lines).

\section{Mobility of screw and edge dislocations}

Once the He configurations have been obtained, the sample yield strength $(\tau)$ for both dislocation types has been studied with a constant $10^{9} \mathrm{~s}^{-1}$ shear strain rate applied to the upper layer of the simulation box in the direction of the Burgers vector.

We analyze the case of the screw dislocation first. The results are plotted in Fig. 5. At $100 \mathrm{~K}$ the stress required for the dislocation to overcome the He bubbles was around $900 \mathrm{MPa}$. The lattice resistance at this temperature as given by the empirical potential used in this study is about $540 \mathrm{MPa}$. The stress to overcome the He bubbles is larger than the lattice resistance stress and therefore the dislocation, once released from the obstacles, will glide in the phonon drag regime. For $300 \mathrm{~K}$, the yield stress was calculated to be around $650 \mathrm{MPa}$, that compares to the lattice resistance of about $180 \mathrm{MPa}$, while at $500 \mathrm{~K}$ the stress required for the dislocation to overcome the He bubbles was about $580 \mathrm{MPa}$ with the lattice resistance regime occurring below $96 \mathrm{MPa}$. Therefore, the required stress for the dislocations to move increases considerably in the presence of He, even at elevated temperatures, resulting in strong hardening.

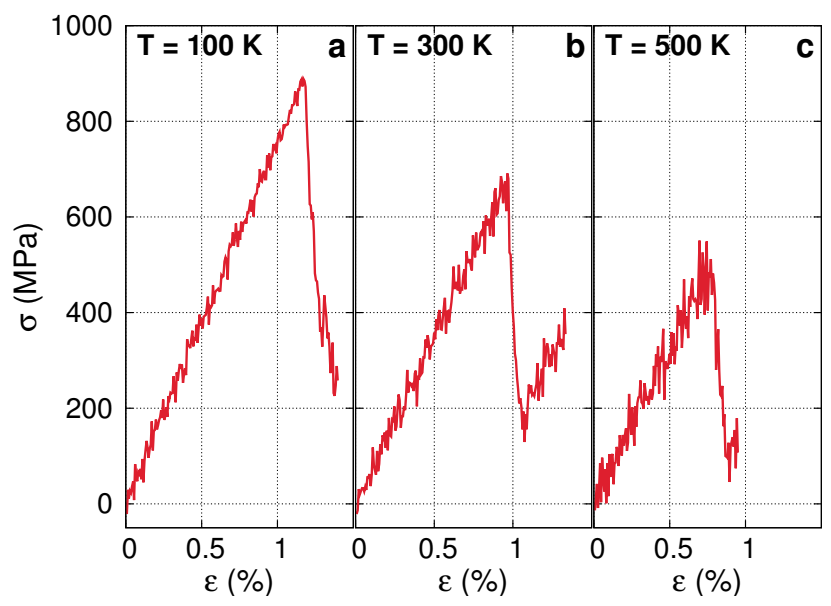

FIG. 5: (Color online) Stress-strain curves for a screw dislocation in $\alpha$-Fe as it overcomes a distribution of $\mathrm{He}$ bubbles at different temperatures (a) $T=100 \mathrm{~K}$; (b)

$T=300 \mathrm{~K}$ and; (c) $T=500 \mathrm{~K}$. The He bubble distributions are the ones obtained using the CMC algorithm.

For the edge dislocations in $\alpha$-Fe gliding on a $\{110\}$ plane it is well known that the Peierls stress is extremely low ${ }^{41,42}$ and their mobility it is often considered athermal. Figure 6 shows the stress-strain curves upon simple shear loading. We observe that He modifies enormously the stress required for the dislocation to move at all temperatures. At $100 \mathrm{~K}$ the yield strength increases up to around $800 \mathrm{MPa}$ before the dislocation overcomes the He bubbles and starts gliding. Increasing the temperature decreases the number of obstacles and increases the probability for the dislocation to move. At $300 \mathrm{~K}$ the yield stress becomes $500 \mathrm{MPa}$ and at $500 \mathrm{~K}$ the stress needed for the dislocation to glide is on the order of 400 MPa.

We highlight here that these results reflect not only the effect of temperature on the bubble-dislocation interaction, but also includes the effects of temperature on the bubble patterning (size and distance between bubbles) that characterizes the He heterogeneous precipitation process.

\section{DISCUSSION}

Traditional models for the yield strength dependence on temperature for a random distribution of obstacles follow the phenomenological relation developed by Kocks et al. ${ }^{43}$ 


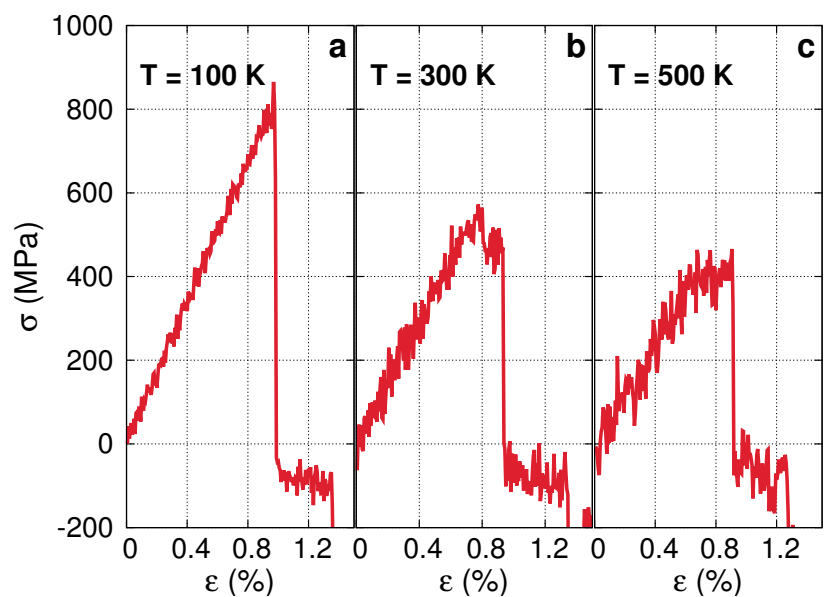

FIG. 6: (Color online) Stress-strain curves for an edge dislocation in $\alpha$-Fe as it overcomes a distribution of $\mathrm{He}$ bubbles at different temperatures (a) $T=100 \mathrm{~K}$; (b)

$T=300 \mathrm{~K}$ and; (c) $T=500 \mathrm{~K}$. The He bubble

distributions are the ones obtained using the CMC algorithm.

$$
\tau=\tau_{0}\left\{1-\left[\frac{T}{T_{0}}\right]^{p}\right\}^{q}
$$

where $\tau_{0}$ is the stress at which the migration barrier for the dislocation becomes zero and $T_{0}$ is the critical temperature at which the thermal energy is high enough for the dislocation to overcome the barrier with no stress applied. In general, $0 \leq p \leq 1$ and $1 \leq q \leq 2$, although their values are somehow arbitrary ${ }^{44}$.

Equation 2 applies to a random distribution of precipitates, distribution that may result from a homogeneous nucleation process. In the case of He precipitating heterogeneously to dislocations we observe that temperature introduces two effects. On one hand, as temperature increases, the bubble diameter increases and their spacing along the dislocation core also increases and, as we shall see, this effect will lead to a non-monotonic relation between stress and temperature. On the other hand, temperature increases the probability of overcoming the Peierls barrier imposed by the lattice resistance. This effect is clearly seen in Fig. 7, where the total yield strength for both screw and edge dislocations is shown in Fig. 7[a] while the yield strength increment with respect to the lattice resistance limit is plotted in Fig. $7[\mathrm{~b}]$. In the first case, Eq. 2 reproduces accurately the values obtained from atomistic calculations with the parameter values shown in Table II. We observe that the values for $p$ and $q$ are indeed within the general range. However, $\tau_{0}$ for the edge dislocation is somehow large. This value is related to the binding energy of the He to the dislocation which is larger for the edge segment than for the screw and therefore the incremental stress to be applied at $0 \mathrm{~K}$ to overcome the barrier is substantially higher.
TABLE II: Fitting parameters for the total yield stress $\tau(T)$.

\begin{tabular}{ccccc}
\hline & $T_{c}(\mathrm{~K})$ & $\tau_{0}(\mathrm{MPa})$ & $p$ & $q$ \\
\hline \hline Screw & 1850 & 1142.88 & 0.53 & 1.04 \\
Edge & 2350 & 7600.03 & 0.04 & 1.06
\end{tabular}

TABLE III: Fitting parameters for the obstacle strengthening $\tau^{*}(T)$.

\begin{tabular}{ccccccc}
\hline & $T_{c}(\mathrm{~K})$ & $\tau_{0}^{*}(\mathrm{MPa})$ & $\alpha$ & $p$ & $q$ & $r$ \\
\hline \hline Screw & 500 & 240 & 1.38 & 2.0 & 1.95 & 1.0 \\
Edge & 2350 & 7600.03 & 0.0 & 0.03 & 0.96 & - \\
\hline
\end{tabular}

The total yield stress includes both the lattice resistance component and the strengthening due to the obstacles. For the edge dislocation the lattice resistance is negligible, while for the screw dislocation there is an important component due to the Peierls barrier in the temperature regime studied in this work ${ }^{45}$. If the lattice resistance for the different temperatures is subtracted from the total stress ${ }^{45}$, the obstacle strengthening is then obtained. The results are shown in Fig. 7[b]. For the edge component the curve is virtually identical to the total stress. On the other hand, for the screw dislocation, we observe a non-monotonic behavior with a maximum at about $350 \mathrm{~K}$. This maximum reflects the fact that the relation between the He bubble distribution, which depends on temperature, and the critical shear stress is not linear. To model this non-monotonic behavior a new term might be added to Eq. 2, rendering the form:

$$
\tau^{*}=\tau_{0}^{*}\left\{1-\left[\frac{T}{T_{0}}\right]^{p}+\alpha\left[\frac{T}{T_{0}}\right]^{r}\right\}^{q}
$$

where the asterisk indicates the component due to obstacle strengthening. A least-square fit to the MD data is shown in Table III and Fig. 7[b].

The quantitative analysis of the results presented here depends on the accuracy of the interatomic potential. More recent $\mathrm{Fe}$ interatomic potentials, like the one by Gordon et $a l^{46}$ or Marinica et $a l^{47}$ that reproduce more accurately the dislocation migration barrier would provide better estimates for the critical shear stress. However, the qualitative description of the various effects to be accounted for under irradiation depending on temperature would not change. The fact that the binding energy of He atoms to the edge dislocation is larger than for the screw would most probably remain the same since the 
atomic density is lower in the edge case (note that this result agrees with previously published trends ${ }^{48}$ ). The effect of dose rate might be more subtle. In a diffusioncontrolled regime the He would have enough time to diffuse and form large bubbles at the dislocation cores. On the other hand, in a reaction-controlled regime He atoms will accumulate quasi-statically at the dislocations without time to migrate and react, therefore remaining disperse at the cores. In an intermediate regime, the relation between the binding energy, the He mobility, the dose rate and the temperature would prescribe the bubble distribution, as shown before, which would depend on the interatomic potential used. This, in turn, would result in a critical shear stress that will differ for each case. Again, the goal of this study is not to obtain perfectly accurate values for the stresses, but to provide a methodology that is able to feed large scale calculations, like crystal plasticity models, to study the macroscopic effect of the formation of bubbles in diverse irradiation environments in an Fe matrix.

Note also that if one is to use the Bacon-Scattergood model ${ }^{49,50}$ that was developed for 'strong' obstacles and that relates the critical shear stress with the harmonic mean of the bubble diameter (D) and spacing $(\mathrm{L})^{51}$ the temperature effect should be taken into account. As a first order approximation, substituting the order of magnitude found above for $\mathrm{D}$ and $\mathrm{L}$ we would obtain:

$$
\tau^{*} \approx \frac{1}{T^{3}}\left\{\ln \left[T^{-1}+T^{-3}\right]^{-1}+\Delta(T)\right\}
$$

which in general decays faster than the results shown in Fig. 7[a] and would present a minimum instead of a maximum if the lattice resistance is subtracted (Fig. 7[b]).

\section{CONCLUSIONS}

In summary, we have studied in this work the role of temperature on the yield stress of screw and edge dislocations in the presence of He bubbles. We have shown, using an object kinetic Monte Carlo methodology, that the distribution of bubbles at a dislocation core depends on both dose rate and temperature, and from these calculations, laws for the diameter and spacing between bubbles were derived, showing the competition between diffusional properties and the particle creation rates. We have studied He segregation to screw and edge straight dislocations in $\alpha$-Fe using an equilibrium Monte Carlo algorithm, including both vibrational and chemical contributions. We observe the formation of bubbles preferentially at the dislocation core in both cases. For a given dose rate the distribution of He atoms at the dislocation cores depends significantly on the temperature. Both approaches, OKMC and CMC, give similar results, supporting the picture that emerges from them in the form of an equation for the size and distance between bubbles.

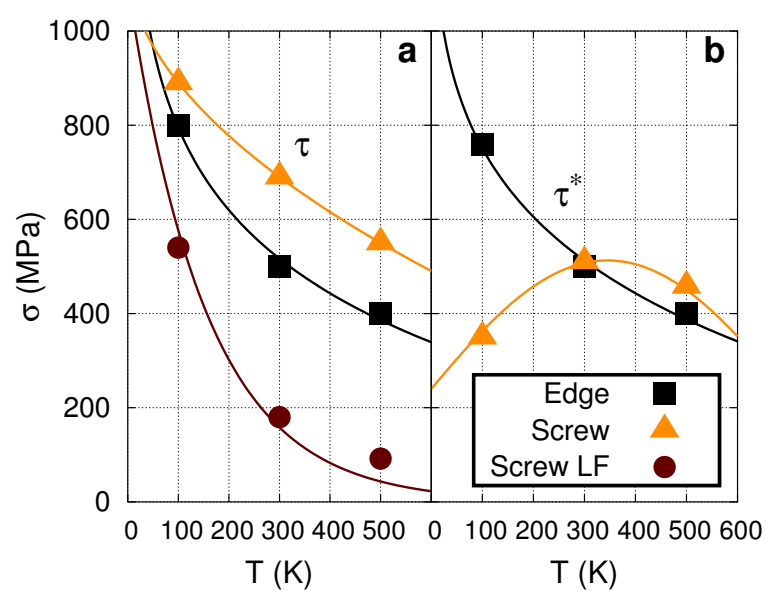

FIG. 7: (Color online) (a) Total yield strength for a screw and an edge dislocations versus temperature for a He concentration of $c_{H e}^{\text {screw }}=3.68 \mathrm{~nm}^{-1}$ and

$c_{H e}^{\text {edge }}=3.57 \mathrm{~nm}^{-1}$. The red circles show the lattice

resistance for a screw dislocation, fitted with an

exponential function. (b) Yield strength versus

temperature for screw and edge dislocations subtracting the lattice resistance for the same He concentration as in (a).

The study of the dislocation mobility shows that $\mathrm{He}$ strongly pins dislocations increasing the system yield strength. Traditional phenomenological Kocks models manage to follow the total critical shear stress given by the atomistic results, if the right set of parameters is chosen. We further show that, if the lattice resistance term is subtracted from the total yield stress, the obstacle strengthening for the screw case results in a nonmonotonic relation, which invalidates these traditional approaches. We have added a new term in Kocks relation of the shear stress with the temperature to be able to reproduce the MD results. The parameters obtained in this study might be used in crystal plasticity calculations to analyze the macroscopic effect of $\mathrm{He}$ in the deformation modes of ferritic systems.

\section{ACKNOWLEDGEMENTS}

The authors want to thank J. Marian and J. P. Hirth for useful discussions. Work performed with support from the Center for Materials at Irradiation and Mechanical Extremes, an Energy Frontier Research Center funded by the U.S. Department of Energy (Award Number 2008LANL1026) at Los Alamos National Laboratory. This research used resources provided by the LANL Institutional Computing Program. LANL, an affirmative action/equal opportunity employer, is operated by Los Alamos National Security, LLC, for the National Nuclear Security Administration of the U.S. DOE under contract DE-AC52-06NA25396. 
* Electronic address: enriquem@lanl.gov

1 N. Baluc, R. Schaublin, P. Spatig, M. Victoria, Nucl. Fusion 44 (2004) 56

${ }^{2}$ R. L. Klueh, D. R. Harris, High-Chromium Ferritic and Martensitic Steels for Nuclear Applications, ASTM MONO3, American Society for Testing and Materials, 2001.

3 M. Gilbert, S. Dudarev, S. Zheng, L. Packer, J.-C. Sublet, Nucl. Fusion 52 (2012) 083019.

${ }^{4}$ D. Kramer, H. R. Brager, C. G. Rhodes, A. G. Pard, J. Nucl. Mater. 25 (1968) 121-131.

${ }^{5}$ U. Stamm, H. Schroeder, J. Nucl. Mater. 155-157 (1988) 1059-1063.

${ }^{6}$ J. Hetherly, E. Martinez, Z. F. Di, M. Nastasi, A. Caro, Scripta Mater. 66 (2012) 17-20.

7 G. R. Odette, D. T. Hoelzer, JOM 62 (9) (2010) 84-92.

8 H. Trinkaus, Radiation Effects: Incorporating Plasma Science and Plasma Technology 101 (1987) 91-107.

9 H. Ullmaier, H. Trinkaus, Materials Science Forum 97-99 (1992) 451-472.

10 H. Ullmaier, J. Chen, J. of Nucl. Mater. 318 (2003) 228233.

11 H. Trinkaus, J. Nucl. Mater. 318 (2003) 234-240.

12 J. Henry, P. Jung, J. Chen, J. C. Brachet, Journal de Physique IV 12 (2002) 103-120.

13 P. Jung, J. Henry, J. Chen, J.-C. Brachet, J. Nucl. Mater. 318 (2003) 241-248.

14 T. Yamamoto, G. R. Odette, H. Hishimoto, J.-W. Rensman, P. Miao, J. of Nucl. Mater. 356 (2006) 27-49.

15 N. N. Kumar, E. Martinez, B. K. Dutta, G. K. Dey, A. Caro, Phys. Rev. B 87 (2013) 054106.

16 S. S. Vagaraly, G. R. Odette, J. Nucl. Mater. 103 (1981) 1239-1243.

17 H. Trinkaus, J. Nucl. Mater. 118 (1983) 39-49.

18 H. Ullmaier, Nucl. Fusion 24 (1984) 1039-1083.

19 R. Schäublin, Y. L. Chiu, J. Nucl. Mater. 362 (2007) 152160

20 S. M. H. Haghighat, R. Schäublin, Phil. Mag. 90 (2010) 1075-1100.

${ }^{21}$ H. L. Heinisch, F. Gao, R. J. Kurtz, Phil. Mag. 90 (2010) $885-895$.

${ }^{22}$ H. L. Heinisch, F. Gao, R. J. Kurtz, J. Nucl. Mater. 367370 (2007) 311-315.

${ }^{23}$ E. M. Bringa, B. D. Wirth, M. J. Caturla, J. Stölken, D. Kalantar, Nucl. Inst. and Meth. in Phys. Research B 202 (2003) 56-63.

${ }^{24}$ L. Zhang, X. Shu, S. Jin, Y. Zhang, G.-G. Lu, J. Phys: Condens. Matter 22 (2010) 375401.

${ }^{25}$ L. Yang, Z. Zhu, S. Peng, X. Long, X. Zhou, X. Zu, H. Heinisch, R. Kurtz, F. Gao, J. Nucl. Mater. 441 (2013) 6-14.

${ }^{26}$ G. Henkelman, H. Jonsson, J. Chem. Phys. 115 (21) (2001)
9657.

27 L. Vernon, S. D. Kenny, R. Smith, E. Sanville, Phys. Rev. B 83 (2011) 075412.

${ }^{28}$ F. El-Mellouhi, N. Mousseau, L. J. Lewis, Phys. Rev. B 78 (2008) 153202.

29 H. Xu, Y. N. Osetsky, R. E. Stoller, Phys. Rev. B 84 (2011) 132103.

30 E. Martìnez, A. Caro, Phys. Rev. B 86 (2012) 214109.

31 D. Frenkel, B. Smit, Understanding Molecular Simulation, Academic Press, 2001.

32 S. Plimpton, Journal of Computational Physics 117 (1995) $1-19$.

33 C. S. Becquart, B. D. Wirth, Comprehensive Nuclear Materials, Elsevier, 2012, Ch. 1.14 Kinetic Monte Carlo Simulations of Irradiation Effects.

${ }^{34}$ W. Cai, Md++. URL http://micro.stanford.edu/MDpp/docs

35 E. Martinez, C.-C. Fu, Phys. Rev. B 84 (2011) 014203.

${ }^{36}$ G. J. Ackland, M. I. Mendelev, D. J. Srolovitz, S. Han, A. V. Barashev, J. Phys: Condens. Matter 16 (2004) 26292642.

37 D. E. Beck, Molecular Physics 14 (1968) 311.

38 N. Juslin, K. Nordlund, J. Nucl. Mater. 382 (2008) 143146.

39 A. Caro, J. Hetherly, A. Stukowski, M. Caro, E. Martinez, S. Srivilliputhur, L. Zepeda-Ruiz, M. Nastasi, J. Nucl. Mater. 418 (2011) 261-268.

40 E. Clouet, Acta Materialia 54 (2006) 3543-3552

41 Y. N. Osetsky, D. Bacon, Modelling Simul. Mater. Sci. Eng. 11 (2003) 427-446.

42 S. Queyreau, J. Marian, M. R. Gilbert, B. D. Wirth, Phys. Rev. B 84 (2011) 064106.

${ }^{43}$ U. F. Kocks, A. S. Argon, M. F. Ashby, Prog. Mat. Sci. 19.

${ }^{44}$ U. F. Kocks, Mater. Sci. Eng. 27 (1977) 291-298.

45 M. R. Gilbert, S. Queyreau, J. Marian, Phys. Rev. B 84 (2011) 174103

46 P. A. Gordon, T. Neeraj, M. I. Mendelev, Phil. Mag. 91 (2011) 3931-3945.

47 L. Proville, D. Rodney, M.-C. Marinica, Nature Materials 11 (2012) 845-849.

48 Y. Zhao, G. Lu, Model. Simul. Mater. Sci. Eng. 19 (2011) 065004.

49 D. J. Bacon, D. M. Barnett, R. O. Scattergood, Prog. Mat. Sci. 23 (1978) 51-262.

50 R. O. Scattergood, D. J. Bacon, Acta Metall. 30 (1982) 1665-1677.

${ }^{51} \tau^{*}=\frac{\mu b}{2 \pi L}\left\{\ln \left[D^{-1}+L^{-1}\right]^{-1}+\Delta\right\}$ where $\mu$ is the shear modulus, $\mathrm{b}$ is the Burgers vector and $\Delta$ is a phenomenological parameter. 